\title{
Near-Infrared Cathodoluminescence Polarimetry of a Plasmonic Vertical Split Ring Resonator
}

Isobel Bicket $^{1}$, Edson Bellido ${ }^{2}$, Sophie Meuret ${ }^{3}$, Toon Coenen ${ }^{4}$, Albert Polman $^{5}$ and Gianluigi Botton ${ }^{6}$

${ }^{1}$ Canadian Centre for Electron Microscopy, McMaster University, Hamilton, Ontario, Canada, Hamilton, Ontario, Canada, ${ }^{2}$ Department of Materials Science and Engineering, McMaster University, Hamilton, ON, Canada, United States, ${ }^{3}$ CEMES-CNRS, 29 Rue Jeanne Marvig, 31055 Toulouse, France, United States, ${ }^{4}$ Delmic BV, Kanaalweg 4, 2628 EB, Delft, The Netherlands, United States, ${ }^{5}$ Center for Nanophotonics, AMOLF, Science Park 104, 1098 XG Amsterdam, The Netherlands, United States, ${ }^{6}$ Department of Materials Science and Engineering, McMaster University, Hamilton, ON, Canada, Canada

Plasmonic nanostructures are characterized by coherent oscillations of conducting charge carriers that are able to interact with light and concentrate electromagnetic (EM) energy into extremely small spaces. In addition, specially designed plasmonic nanoparticles are able to interact selectively with different polarizations of light, promoting plasmonic devices as platforms for enhancing signal in linear or circular dichroism spectroscopies, or enhancing selectivity in reactions of chiral molecules in the near-infrared (NIR) or visible regions of the spectrum [1,2]. Net dichroic signal enhancement can be achieved with nanostructures which support degenerate electric and magnetic dipole moments [3], such as the split ring resonator (SRR).

The lowest energy resonance mode of an SRR supports a magnetic dipole orthogonal to an electric dipole [4]. SRRs are relatively easy to fabricate flat on a substrate, but in this orientation the magnetic dipole is oriented along the substrate normal and is difficult to couple to incoming light [5]. Fabricating a vertical SRR (VSRR) is more challenging, but a VSRR supports an in-plane magnetic dipole and an in-plane electric dipole, both of which can be coupled simultaneously to far-field radiation [6].

We have fabricated VSRRs with a base length of approximately $280 \mathrm{~nm}$ using two successive electron beam lithography patterning and deposition steps, on a TEM-compatible SiNx membrane. Because of the fabrication parameters used, the VSRRs have hollow pillars. The solidity of the pillars is expected to affect the higher order resonances, which have higher frequency spatial fluctuations in charge density, more than the lower order resonances. We then performed polarized cathodoluminescence (CL) spectroscopy on the NIR and visible plasmonic resonances supported in our fabricated VSRRs.

CL data was acquired on an FEI XL-30 field emission SEM in the NIR and visible regions of the spectrum, using the acquisition set-up shown schematically in Fig. 1(a). The polarization properties of the emission are described using the Stokes parameters, which are calculated from six experimental datasets acquired with different settings of a quarter wave plate (QWP) and linear polarizer (LP) [7]. To account for possible fluctuations in beam current, the intensity of the CL emission was scaled by a factor relative to the simultaneous signal from the secondary electron detector in the SEM. In order to overcome the challenges of low emission efficiency and poor detector response in the NIR, we insert a long-pass filter of $980 \mathrm{~nm}$ into the optical path before integrating all of the remaining NIR light on only a few channels in the spectrometer. This acquisition technique loses all NIR spectral sensitivity, but boosts the signal-to-noise ratio, enabling us to acquire enough signal with approximately $7.1 \mathrm{nA}$ of beam current and an integration time of $2.5 \mathrm{~s} / \mathrm{pixel}$.

Simulations of the far-field emission under electron beam irradiation were performed using the MNPBEM Matlab® toolbox [8]. Analysis of the electric fields from these simulations and analytical calculations of model dipole moments were performed using custom Python code [9]. Simulations show that the far-field emission from the lowest energy peak of the VSRR can be fitted using a simple pair of dipole moments: an electric dipole aligned along the long axis of the VSRR, and a magnetic dipole directed through the gap between the 
resonator pillars (Fig. 1(b)). These simulations reveal that while the emission is dominated by linearly polarized light, the overlap of the magnetic and electric dipoles results in a significant amount of circular polarization in the high angle far-field emission (i.e.: S3 in Fig. 1(b)).

Electron energy loss spectroscopy on our fabricated VSRR, performed in a monochromated FEI Titan STEM, confirms the presence of a single peak in the NIR at approximately $1800 \mathrm{~nm}$ in wavelength $(0.7 \mathrm{eV})$ (Fig. 1(c)), followed by a series of closely spaced higher order multipole resonances around the rims of the VSRR pillars below $\sim 900 \mathrm{~nm}$ (above $\sim 1.35 \mathrm{eV}$ ) [10]. The EELS data justifies the NIR integration used to acquire CL data on the dipole mode. The NIR CL data (Fig. 1(d)) shows that the collected emission is dominantly linearly polarized along the long axis of the VSRR, in agreement with simulations and analytical calculations. In addition, a non-negligible amount of circularly polarized light is collected by the mirror-slit combination as the electron beam is scanned across the sample, suggesting an interesting relationship between near-field beamsample interactions, and the far-field emission from the sample [11]. The polarization response of the VSRR can then be further extrapolated to predict its usefulness as a platform for dichroic spectroscopy or interactions with chiral molecules. We suggest that the techniques used in this study may be further useful towards evaluating the potential of other plasmonic nanostructures in dichroic experiments.

Acknowledgements: The experimental EELS work was performed in the Canadian Centre for Electron Microscopy and is supported by the Natural Sciences and Engineering Research Council of Canada (NSERC) under the Discovery Grant Program (ICB, EPB, GAB). Experimental CL work was performed at AMOLF, which is part of the research program of the Netherlands Organization for Scientific Research and is funded by the European Research Council (ICB, SM, TC, AP).

(a)
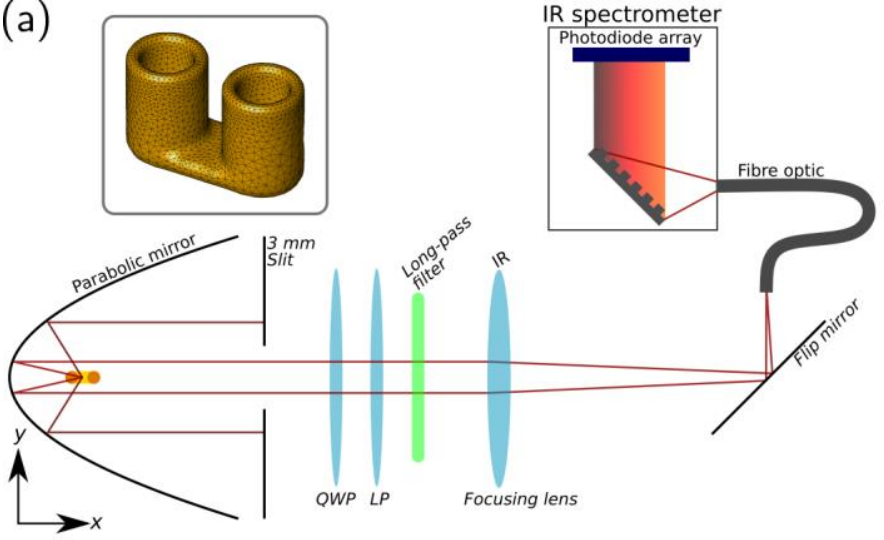

(c)
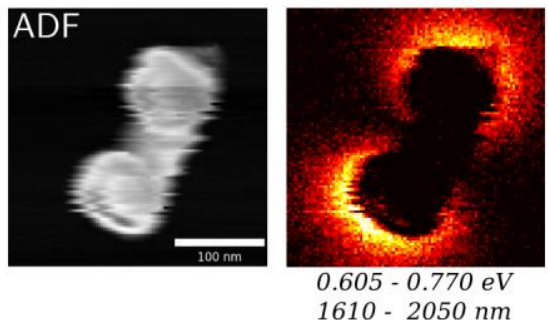

(d)

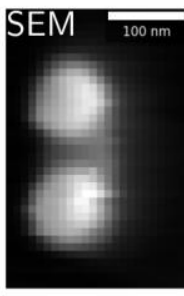

(b)
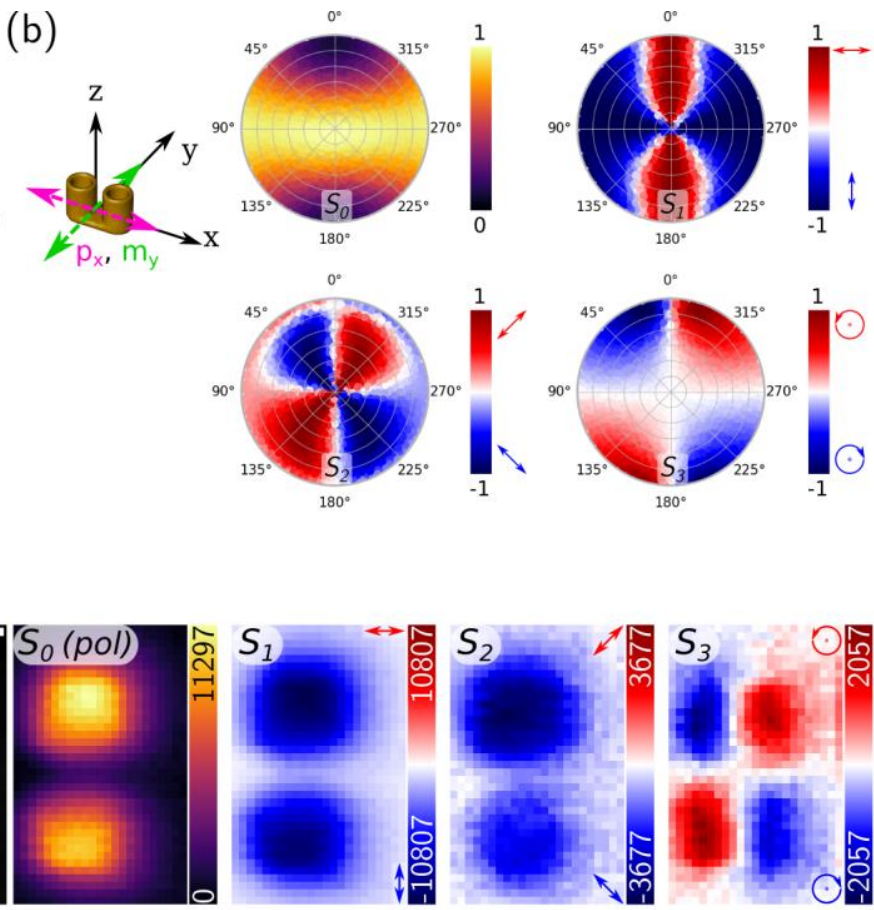

Figure 1. (a) Schematic of the experimental CL acquisition set-up: briefly, the sample is excited by an electron beam and may emit light. The light is collected by a mirror, filtered by a $3 \mathrm{~mm}$ wide slit, a QWP, LP, and then a long-pass filter, before being focused onto an IR spectrometer. A model of the VSRR with hollow pillars is shown in the inset. (b) Analytical calculations of the electric fields emitted from the combination of a magnetic dipole (green) and an orthogonal electric dipole (pink), as fitted to the simulated emission of the VSRR. Arrows 
by the colourbars indicate the polarization direction of light relative to the $(\theta, \varphi)$ polar axes. (c) ADF-STEM image (left) of a VSRR and EELS map (right) of the NIR dipole mode. (d) CL polarimetry results on a VSRR: SEM image (left) and the four Stokes parameters calculated from six NIR datasets; arrows indicate the polarization direction of light relative to the Cartesian image coordinates for each Stokes parameter.

\section{References}

[1] J. T. Collins et al., Advanced Optical Materials, 5, 16, 1700182, 2017.

[2] Y. Tang and A. E. Cohen, Phys. Rev. Lett., 104, 16, 163901, Apr. 2010.

[3] A. García-Etxarri and J. A. Dionne, Phys. Rev. B, 87, 23, 235409, Jun. 2013.

[4] S. Mühlig et al., Metamaterials, 5, 2, 64-73, Jun. 2011.

[5] P. Gay-Balmaz and O. J. F. Martin, J. Appl. Phys., 92, 5, 2929-2936, Sep. 2002.

[6] P. C. Wu et al., Sci. Rep., 5, p. 9726, Jun. 2015.

[7] C. I. Osorio et al., ACS Photonics, 3, 1, 147-154, Jan. 2016.

[8] U. Hohenester, Comput. Phys. Commun., 185, 3, 1177-1187, Mar. 2014.

[9] I.C. Bicket (2020), "CLFields" [Python 3], https://github.com/icbicket/CLFields. DOI: 10.5281/zenodo.3739563

[10] I. C. Bicket, et al., Microscopy, 67, suppl_1, i40-i51, Mar. 2018.

[11] F.-P. Schmidt et al., Nano Lett., 21, 1, 590-596, Jan. 2021. 